\title{
Nhân tố ảnh hưởng đến tính hiệu lực của hệ thống kiểm soát nội bộ hoạt động tín dụng tại ngân hàng thương mại Việt Nam
}

\section{Factors impact on the effectiveness of internal control systems in credit facilities in Vietnam commercial banks}

\author{
Nguyễn Thị Loan ${ }^{1 *}$ \\ ${ }^{1}$ Trường Đại học Ngân hàng Thành phố Hồ Chí Minh, Việt Nam \\ *Tác giả liên hệ, Email: loan.dhnh@gmail.com
}

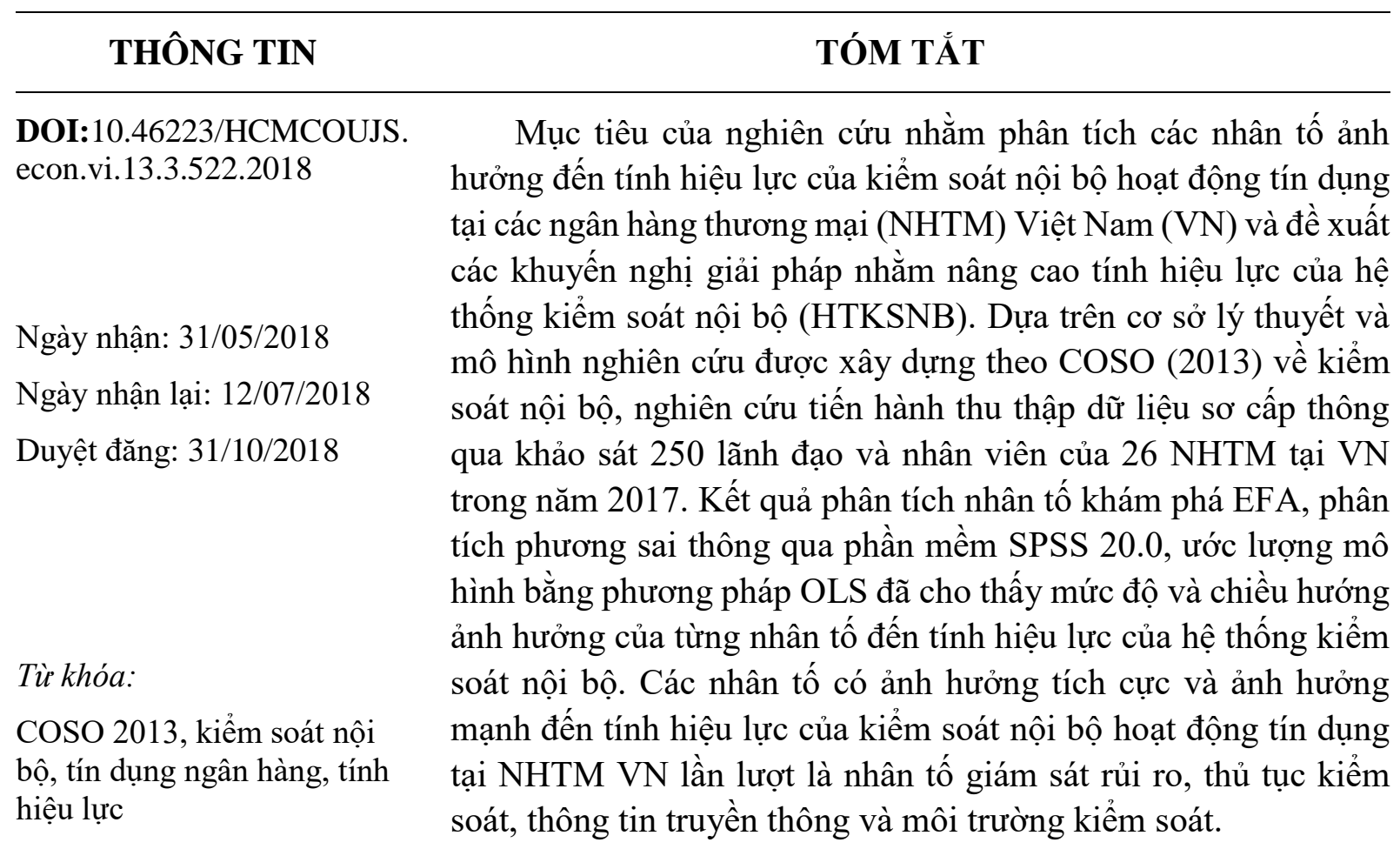

\section{ABSTRACT}

The study aims to analyse factors that affect the effectiveness of internal control systems in credit facilities in Vietnam commercial banks and we suggest some solutions to improve the effectiveness of internal control systems. The study used the theories and integrated framework by COSO (2013) and it was carried out for collecting data from 250 managers and employees of 26 commercial banks in Vietnam in 2017. Using exploratory factor analysis (EFA), factor analysis in SPSS Statistics 20.0, Keywords:

bank credit, COSO Internal Control (2013), internal control, the effectiveness forecasting models by OLS method, the findings showed that the degree and bias of each factor affect the effectiveness of internal control systems. The factors of risk assessment, control activities, 
information and communication, and the control environment will all work to the degree of effectiveness of internal control systems in Vietnam.

\section{Giới thiệu}

Trong các hoạt động kinh doanh tại các NHTM VN, hoạt động tín dụng là hoạt động tạo ra lợi nhuận cao nhất đối với phần lớn ngân hàng (Báo cáo tài chính của NHTM Việt Nam), tuy nhiên cũng là hoạt động tiềm ẩn rủi ro cao nhất. Với sự hội nhập quốc tế ngày càng sâu rộng của VN hiện nay, Chính phủ đã dần nới lỏng các quy định kiểm soát đối với hoạt động ngân hàng, có sự gia tăng cạnh tranh và gia tăng ảnh hưởng của tình hình kinh tế thế giới và khu vực đối với hoạt động kinh doanh của các NHTM làm cho rủi ro trong hoạt động kinh doanh nói chung và rủi ro tín dụng nói riêng ngày càng gia tăng. Trong khi đó, vẫn còn những NHTM VN đang trong quá trình tái cơ cấu, giải quyết vấn đề nợ xấu. Vì vậy, nghiên cứu về KSNB của COSO và vận dụng để phân tích nhân tố tác động đến tính hiệu lực của kiểm soát nội bộ đối với hoạt động tín dụng tại các NHTM VN là cần thiết, có ý nghĩa cả về lí luận và thực tiễn. Về mặt lí luận, khẳng định các nhân tố và đưa ra bằng chứng thực nghiệm về sự tác động của các nhân tố đến tính hiệu lực của hệ thống KSNB hoạt động tín dụng tại NHTM VN. Về mặt thực tiễn, hướng đến gợi ý các chính sách có tính khả thi đối với NHTM góp phần nâng cao tính hiệu lực đối với kiểm soát hoạt động tín dụng tại ngân hàng.

\section{Cơ sở lí thuyết về KSNB theo COSO và tổng quan nghiên cứu trước}

COSO (Committee of Sponsoring Organization) là Ủy ban thuộc Hội đồng quốc gia Hoa Kì về việc chống gian lận về báo cáo tài chính, là tổ chức nghiên cứu, thống nhất và công bố hệ thống kiểm soát nội bộ. Với sự thay đổi rất lớn của môi trường hoạt động kinh doanh trong hơn 20 năm qua, Coso đã ban hành Khuôn mẫu thống nhất về HTKSNB vào tháng 5/2013, cập nhật và thay thế khuôn mẫu thống nhất về HTKSNB năm 1992, cho phù hợp với điều kiện môi trường kinh doanh ngày càng phức tạp, công nghệ phát triển và toàn cầu hóa. Theo đó, "Kiểm soát nội bộ là một quá trình, chịu ảnh hưởng bởi hội đồng quản trị, người quản lí và các nhân viên của đơn vị, được thiết kế để cung cấp một sự đảm bảo hợp lí nhằm đạt được các mục tiêu về hoạt động hiệu quả, báo cáo tin cậy và tuân thủ quy định" (COSO, 2013).

Đối với hoạt động tín dụng thì kiểm soát nội bộ phải đảm bảo hợp lý để vừa đạt các mục tiêu tín dụng có hiệu quả, vừa đảm bảo mức độ tin cậy của các thông tin báo cáo và tuân thủ pháp luật, các chính sách, các quy định.

Theo khuôn mẫu lí thuyết về KSNB trong báo cáo COSO (1992), HTKSNB gồm 5 thành phần (nhân tố) là môi trường kiểm soát $(\mathrm{MT})$, đánh giá rủi ro $(\mathrm{ĐG})$, thủ tục kiểm soát (TTKS), thông tin truyền thông (TT) và giám sát (GS). Mô hình KSNB theo khuôn mẫu COSO năm 2013 vẫn gồm 5 nhân tố trên, nhưng có bổ sung thêm 17 nguyên tắc (Bảng 1). 


\section{Bảng 1}

Các nhân tố và nguyên tắc ảnh hưởng đến Hệ thống kiểm soát nội bộ theo COSO (2013)

\begin{tabular}{|c|c|c|}
\hline STT & $\begin{array}{c}\text { Nhân tố } \\
\text { HTKSNB }\end{array}$ & Nguyên tắc \\
\hline 1 & $\begin{array}{l}\text { Môi trường kiểm } \\
\text { soát }\end{array}$ & $\begin{array}{l}\text { 1. Cam kết về tính trung thực và tuân thủ giá trị đạo đức } \\
\text { 2. Chịu trách nhiệm giám sát } \\
\text { 3. Thiết lập cơ cấu quyền hạn và trách nhiệm } \\
\text { 4. Thực thi cam kết về năng lực } \\
\text { 5. Đảm bảo trách nhiệm giải trình }\end{array}$ \\
\hline 2 & Đánh giá rủi ro & $\begin{array}{l}\text { 6. Các mục tiêu phù hợp và cụ thể } \\
\text { 7. Xác định và phân tích rủi ro } \\
\text { 8. Đánh giá rủi ro gian lận } \\
\text { 9. Nhận diện và phân tích các thay đổi trọng yếu }\end{array}$ \\
\hline 3 & $\begin{array}{l}\text { Hoạt động kiểm } \\
\text { soát }\end{array}$ & $\begin{array}{l}\text { 10. Lựa chọn và phát triển hoạt động kiểm soát } \\
\text { 11. Lựa chọn và phát triển hoạt động kiểm soát chung về công } \\
\text { nghệ } \\
\text { 12. Triển khai thực hiện thông qua chính sách và thủ tục kiểm } \\
\text { soát }\end{array}$ \\
\hline 4 & $\begin{array}{l}\text { Thông tin và } \\
\text { truyền thông }\end{array}$ & $\begin{array}{l}\text { 13. Sử dụng các thông tin thích đáng phù hợp } \\
\text { 14. Truyền thông nội bộ } \\
\text { 15. Truyền thông bên ngoài tổ chức }\end{array}$ \\
\hline 5 & $\begin{array}{l}\text { Hoạt động giám } \\
\text { sát }\end{array}$ & $\begin{array}{l}\text { 16. Thực hiện hoạt động đánh giá thường xuyên hoặc định kỳ } \\
\text { 17. Đánh giá và truyền thông báo cáo giám sát }\end{array}$ \\
\hline
\end{tabular}

Nguồn: COSO (2013)

Tính hiệu lực của HTKSNB là sự hoạt động theo các quy định liên quan đến 5 nhân tố trong KSNB (Ayagre, Appiah-Gyamerah \& Nartey, 2014). Đánh giá HTKSNB có hiệu lực và hiệu quả hay không là phụ thuộc kết quả đánh giá sự hoạt động của 5 nhân tố của KSNB (COSO, 2013). Đây cũng chính là các nhân tố sẽ ảnh hưởng đến tính hiệu lực và hiệu quả của KSNB nói chung và KSNB hoạt động tín dụng nói riêng.

Cùng quan điểm với COSO, HTKSNB hiệu lực và hiệu quả là một thành phần quan trọng của quản trị ngân hàng $(\mathrm{NH})$ và là nền tảng cho $\mathrm{NH}$ hoạt động an toàn, đảm bảo đạt mục tiêu đặt ra (Basel, 1998). Ủy ban Basel về giám sát $\mathrm{NH}$ qua khảo sát về các thất bại lớn và những vụ sụp đổ của các ngân hàng trên thế giới cũng đã kết luận một trong các nguyên nhân chủ yếu đó là sự thất bại của ban lãnh đạo $\mathrm{NH}$ trong việc thiết lập và duy trì một HTKSNB hiệu 
lực và hiệu quả. Các nghiên cứu ở các quốc gia trên thế giới cũng đưa đến kết luận HTKSNB hiệu lực và hiệu quả sẽ ngăn ngừa và phát hiện các gian lận và sai sót trong hoạt động ngân hàng (Abiola \& Oyewole, 2013; Ajala, Amuda \& Arulogun, 2013; Olatunji, 2009; Salehi, Shiri \& Ehsanpour, 2013). Điều này cho thấy tầm quan trọng của hệ thống KSNB đối với hoạt động kinh doanh của ngân hàng.

Đã có các nghiên cứu quốc tế vận dụng lí thuyết của COSO đánh giá tính hữu hiệu của HTKSNB tại ngân hàng. Điển hình như Sultana và Haque (2011), với phương pháp khảo sát 6 NH tư nhân tại Bangladesh, sử dụng thang đo Likert đánh giá 5 nhân tố cấu thành HTKSNB theo COSO với 3 mục tiêu hiệu quả, thông tin tin cậy và tuân thủ, kết quả cho thấy HTKSNB tại $6 \mathrm{NH}$ này có hiệu quả, mục tiêu KSNB về tuân thủ được đáp ứng cao nhất. Nghiên cứu của Ayagre và cộng sự (2014), khảo sát có sử dụng thang đo Likert và phần mềm SPSS, phạm vi tại các NH ở Ghana, tiếp cận 5 nhân tố cấu thành HTKSNB theo COSO (2013), kết quả HTKSNB tại các NH ở Ghanaian tương đối tốt, nhân tố môi trường kiểm soát và giám sát hoạt động được đánh giá cao, điểm trung bình 4,72 và 4,66 . Salehi và cộng sự (2013) cũng sử dụng bảng câu hỏi với thang đo Likert nghiên cứu ảnh hưởng của HTKSNB của NH Mellat ở Iran trong việc ngăn ngừa các gian lận và sai sót. Kết quả nghiên cứu cho thấy mỗi thành phần trong HTKSNB càng yếu kém thì khả năng sai sót và gian lận càng nhiều, trong đó môi trường kiểm soát tốt sẽ góp phần nhiều nhất trong việc ngăn ngừa gian lận và sai sót.

Barakat (2009) sử dụng phương pháp khảo sát các NH tại Jordan với 41 câu hỏi để đánh giá 5 nhân tố cấu thành HTKSNB theo Basel II bao gồm tầm nhìn quản trị và văn hóa lãnh đạo; đánh giá rủi ro; hoạt động kiểm soát; thông tin và truyền thông; giám sát hoạt động và sửa chữa sai sót, phần lớn các nhân tố được đánh giá cao so với mức điểm trung bình. Trong 5 nhân tố trên, nhân tố giám sát hoạt động, sửa chữa sai sót và nhận diện, đánh giá rủi ro là yếu nhất, cần được đặc biệt quan tâm để nâng cao hiệu quả KSNB. Olatunji (2009) khảo sát 50 NH tại Nigeria để nghiên cứu có hay không mối quan hệ giữa kiểm soát nội bộ và gian lận, kết quả cho thấy gian lận thâm nhập vào ngân hàng gây tổn thất lớn, kéo lùi sự phát triển của hệ thống tài chính và tác giả đã đề xuất giải pháp xây dựng HTKSNB chặt chẽ, kiểm toán nội bộ hiệu quả, quản lí tiền mặt sâu sát, phân công rõ ràng, cải tiến chính sách nhân sự, tuyển dụng.

Tại Việt Nam, P. M. Nguyen (2014) phân tích một số yếu kém trong hoạt động kiểm soát đối với nghiệp vụ tín dụng; đánh giá về KSNB của các NHTM VN so với các tiêu chuẩn quốc tế của Coso hay Basel (Pham \& Nguyen, 2015; Vo \& Le, 2014), nghiên cứu về những lí thuyết liên quan đến KSNB trong NHTM theo tiêu chuẩn của Basel hoặc Coso (Ngo \& Le, 2015 ; Vo, 2015); Dao và Le (2012) nghiên cứu về KSNB gắn với rủi ro trong hoạt động $\mathrm{NH}$; P. A. Nguyen và Ha (2010) xây dựng mô hình các nhân tố tác động đến hiệu quả hoạt động KSNB các NHTM trên địa bàn Thành phố Hồ Chí Minh dựa trên 13 nguyên tắc về KSNB theo ủy ban Basel về giám sát $\mathrm{NH}$. Tuy nhiên, theo nghiên cứu chưa đầy đủ của tác giả bài viết, các nghiên cứu về KSNB hoạt động tín dụng tại NHTM còn khá khiêm tốn, chủ yếu là các nghiên cứu về KSNB nói chung, chưa nghiên cứu đánh giá cụ thể về các nhân tố tác động đến tính hữu hiệu của KSNB hoạt động tín dụng tại NH. 


\section{Mô hình và phương pháp nghiên cứu}

Dựa vào cơ sở lí thuyết của COSO và nghiên cứu của Ayagre và cộng sự (2014), bài viết tiếp cận mô hình nghiên cứu gồm 5 nhân tố cấu thành HTKSNB theo COSO (2013) kết hợp với sử dụng thang đo Likert và phần mềm SPSS, nghiên cứu có mô hình hồi quy bội mô tả mối quan hệ giữa các nhóm nhân tố (biến độc lập) đối với biến phụ thuộc được biểu diễn:

$$
H H i=\beta 0+\beta 1 M T i+\beta 2 D G i+\beta 3 T T K S i+\beta 4 T T i+\beta 5 G S i+\varepsilon
$$

Trong đó: HHi (Tính hiệu lực của HTKSNB tín dụng) là biến phụ thuộc, và MT, DG, TTKS, TT, GS là các biến độc lập liên quan đến 5 nhân tố môi trường kiểm soát, đánh giá rủi ro, thủ tục kiểm soát, thông tin và truyền thông, giám sát rủi ro trong lí thuyết của COSO.

Dựa vào giả thiết nghiên cứu của Ayagre và cộng sự (2014), bài viết đã kế thừa và đặt giả thuyết nghiên cứu để phục vụ cho quá trình phân tích dữ liệu liên quan đến các yếu tố ảnh hưởng đến tính hiệu lực của HTKSNB tín dụng tại ngân hàng:

H1: Môi trương kiểm soát có ảnh huởng tích cục đến tính hiệu lục của HTKSNB hoạt động TD tại $\mathrm{NH}$

H2: Đánh giá rủi ro có ảnh hương tích cục đến tính hiệu lục của HTKSNB hoạt động TD tại NH

H3: Thủ tục kiểm soát có ảnh hương tích cực đến tính hiệu lục của HTKSNB hoạt động TD tại NH

H4: Chất luợng hệ thống thông tin và truyền thông có ảnh hưởng tích cực đến tính hiệu lục của HTKSNB hoạt động TD tại NH

H5: Hoạt động giám sát có ảnh huởng tích cực đến tính hiệu lục của HTKSNB hoạt động TD tại $N H$

Các giả thiết được đặt ra là cơ sở để nghiên cứu đánh giá mức độ tác động của các nhân tố (biến phụ thuộc) ảnh hưởng đến tính hiệu lực của HTKSNB hoạt động tín dụng (biến độc lập), và các nhân tố đều được giả thiết là có tác động tích cực (tác động dương, cùng chiều) với tính hiệu lực của HTKSNB hoạt động tín dụng tại NHTM.

Nghiên cứu đã thực hiện gửi bảng khảo sát trực tiếp, sau khi loại các phiếu trả lời không đủ thông tin, thu về được 250 mẫu khảo sát với đầy đủ thông tin từ lãnh đạo và nhân viên của 26 NHTM tại VN (khảo sát từ tháng 8/2017 đến tháng 12/2017). Bảng câu hỏi khảo sát bao gồm 50 mục hỏi được xây dựng kết hợp dựa theo các nghiên cứu của Ayagre và cộng sự (2014), công cụ đánh giá tính hiệu lực của KSNB theo báo cáo COSO (2013), các nguyên tắc của Basel và kết hợp các quy định về HTKSNB tín dụng tại các NHTM tại VN. Phân bổ các câu hỏi khảo sát (Bảng 2) với thang đo Likert 5 mức độ để phân tích tính hiệu lực của các quy định nội bộ về kiểm soát nội bộ đối với hoạt động tín dụng tại ngân hàng. Bảng câu hỏi với thang đo từ 1 Rất thấp, 2 - Thấp, 3 - Trung bình, 4 - Cao và 5 - Rất cao, từ đó vận dụng mô hình định lượng để phân tích các nhân tố ảnh hưởng đến tính hiệu lực của HTKSNB hoạt động tín dụng tại NHTM. Bên cạnh các câu hỏi khảo sát với thang đo Likert 5 , nghiên cứu còn có thiết kế các câu hỏi mở cuối Bảng khảo sát để tổng hợp các ý kiến của lãnh đạo, nhân viên NHTM về các 
giải pháp tác động đến các nhân tố của HTKSNB tín dụng góp phần nâng cao tính hiệu lực trong kiểm soát nội bộ trong hoạt động tín dụng để có cơ sở kiến nghị giải pháp.

\section{Bảng 2}

Thống kê câu hỏi khảo sát

\begin{tabular}{|c|l|c|c|}
\hline STT & \multicolumn{1}{|c|}{ Nội dung câu hỏi } & Số lượng & Thứ tụ̣ \\
\hline 1 & Phần thông tin chung & 9 & Q1 - Q9 \\
\hline 2 & Môi trường kiểm soát (MT) & 9 & Q10 - Q18 \\
\hline 3 & Đánh giá rủi ro (ĐG) & 5 & Q19 - Q23 \\
\hline 4 & Thủ tục kiểm soát (TTKS) & 9 & Q24 - Q32 \\
\hline 5 & Thông tin và truyền thông (TT) & 7 & Q33 - Q39 \\
\hline 6 & Hoạt động giám sát (GS) & 7 & Q40 - Q46 \\
\hline 7 & Tính hiệu lực hệ thống KSNB (HH) & 4 & Q47 - Q50 \\
\hline
\end{tabular}

Tổng cộng

50 câu hỏi

Nguồn: Kết quả phân tích dữ liệu của nhóm nghiên cứu

\section{Bảng 3}

Khảo sát nhân tố ảnh hưởng đến tính hiệu lực Hệ thống KSNB TD tại NHTM

\begin{tabular}{|c|c|c|c|c|c|c|}
\hline STT & Diễn giải & $\begin{array}{l}\text { (1) } \\
\text { Rất } \\
\text { thấp }\end{array}$ & $\begin{array}{c}\text { (2) } \\
\text { Thấp }\end{array}$ & $\begin{array}{c}\text { (3) } \\
\text { Trung } \\
\text { bình }\end{array}$ & $\begin{array}{l}\text { (4) } \\
\text { Cao }\end{array}$ & $\begin{array}{l}\text { (5) } \\
\text { Rất } \\
\text { cao }\end{array}$ \\
\hline \multicolumn{7}{|c|}{ Nhân tố "Môi trường kiểm soát"- MT } \\
\hline MT 1 & $\begin{array}{l}\text { Lãnh đạo NH chấp hành tốt các quy } \\
\text { định kiểm soát tín dụng tại ngân hàng }\end{array}$ & $\square$ & $\square$ & $\square$ & $\square$ & $\square$ \\
\hline MT2 & $\begin{array}{l}\text { NH có chính sách tuyển dụng nhân viên } \\
\text { và nhân viên tín dụng rõ ràng. }\end{array}$ & $\square$ & $\square$ & $\square$ & $\square$ & $\square$ \\
\hline MT3 & $\begin{array}{l}\text { NH có kế hoạch cụ thể, rõ ràng chính } \\
\text { sách đào tạo đối với lãnh đạo và nhân } \\
\text { viên. }\end{array}$ & $\square$ & $\square$ & $\square$ & $\square$ & $\square$ \\
\hline MT4 & $\begin{array}{l}\text { NH có chính sách lương, thưởng, kỷ } \\
\text { luận rõ ràng, cụ thể }\end{array}$ & $\square$ & $\square$ & $\square$ & $\square$ & $\square$ \\
\hline
\end{tabular}




\begin{tabular}{|c|c|c|c|c|c|c|}
\hline STT & Diễn giải & $\begin{array}{l}\text { (1) } \\
\text { Rất } \\
\text { thấp }\end{array}$ & $\begin{array}{c}\text { (2) } \\
\text { Thấp }\end{array}$ & $\begin{array}{l}\text { (3) } \\
\text { Trung } \\
\text { bình }\end{array}$ & $\begin{array}{l}\text { (4) } \\
\text { Cao }\end{array}$ & $\begin{array}{l}\text { (5) } \\
\text { Rất } \\
\text { cao }\end{array}$ \\
\hline MT5 & $\begin{array}{l}\text { NH có chính sách cụ thể vể luân chuyển } \\
\text { cán bộ, nhân viên theo định kỳ }\end{array}$ & & & & L & \\
\hline MT6 & $\begin{array}{l}\text { NH có quy định cụ thể và thể chế hóa } \\
\text { bằng văn bản rõ ràng chức năng, trách } \\
\text { nhiệm của cán bộ quản lý và từng nhân } \\
\text { viên. }\end{array}$ & & & & & \\
\hline MT7 & $\begin{array}{l}\text { Cẩm nang về đạo đức nghề nghiệp được } \\
\text { phổ biến nhắc lại và có cam kết của } \\
\text { nhân viên từng định kỳ }\end{array}$ & & & & \begin{tabular}{|l} 
\\
\end{tabular} & \\
\hline MT8 & $\begin{array}{l}\text { Bộ phận kiểm soát nội bộ thực hiện } \\
\text { được chức năng kiểm soát rủi ro tín } \\
\text { dụng tại ngân hàng }\end{array}$ & & & & & \\
\hline MT9 & $\begin{array}{l}\text { Trình độ, năng lực chuyên môn của } \\
\text { kiểm toán nội bộ và bộ phận kiểm tra, } \\
\text { kiểm soát nội bộ NH }\end{array}$ & & & & & \\
\hline \multicolumn{7}{|c|}{ Nhân tố "Đánh giá rủi ro" - ĐG } \\
\hline ĐG1 & $\begin{array}{l}\text { Quy trình soát xét chất lượng tín dụng } \\
\text { có khả năng dự báo sớm những thay đồi } \\
\text { về tình hình tài chính, khả năng trả nợ } \\
\text { của các bên đối tác }\end{array}$ & & & & & \\
\hline ĐG2 & $\begin{array}{l}\text { Tính kịp thời của các thông tin cảnh báo } \\
\text { rủi ro khi có sự thay đổi bất lợi trong } \\
\text { môi trường kinh doanh, hoạt động tín } \\
\text { dụng }\end{array}$ & & & & & \\
\hline ĐG3 & $\begin{array}{l}\text { Tính cập nhật của các quy định về } \\
\text { ngành nghề kinh doanh, quản lý rủi ro } \\
\text { tín dụng }\end{array}$ & & & & & \\
\hline ĐG4 & $\begin{array}{l}\text { Mức độ linh hoạt về lãi suất tín dụng đối } \\
\text { với với KH trên cơ sở phân loại khách } \\
\text { hàng khi xem xét cấp tín dụng }\end{array}$ & & & & & \\
\hline ĐG5 & $\begin{array}{l}\text { NH có các tiêu chí cảnh báo sớm nợ có } \\
\text { vấn đề }\end{array}$ & & & & Г & $\square$ \\
\hline
\end{tabular}




\begin{tabular}{|c|c|c|c|c|c|c|}
\hline STT & Diễn giải & $\begin{array}{l}\text { (1) } \\
\text { Rất } \\
\text { thấp }\end{array}$ & $\begin{array}{c}\text { (2) } \\
\text { Thấp }\end{array}$ & $\begin{array}{l}\text { (3) } \\
\text { Trung } \\
\text { bình }\end{array}$ & $\begin{array}{l}(4) \\
\text { Cao }\end{array}$ & $\begin{array}{l}\text { (5) } \\
\text { Rất } \\
\text { cao }\end{array}$ \\
\hline \multicolumn{7}{|c|}{ Nhân tố "Thủ tục kiểm soát" - TTKS } \\
\hline $\begin{array}{l}\text { TTK } \\
\text { S1 }\end{array}$ & $\begin{array}{l}\text { Tính hiệu lực trong kiểm soát tín dụng } \\
\text { từ thực hiện theo quy trình tín dụng nội } \\
\text { bộ NH. }\end{array}$ & & & & ${ }^{-}$ & \\
\hline $\begin{array}{l}\text { TTK } \\
\text { S2 }\end{array}$ & $\begin{array}{l}\text { Tính hiệu lực trong kiểm soát tín dụng } \\
\text { từ thực hiện theo quy định nội bộ về xếp } \\
\text { hạng tín dụng đối với khách hàng doanh } \\
\text { nghiệp }\end{array}$ & & & & & \\
\hline $\begin{array}{c}\text { TTK } \\
\text { S3 }\end{array}$ & $\begin{array}{l}\text { Tính hiệu lực trong kiểm soát tín dụng } \\
\text { từ thực hiện theo quy định nội bộ về xếp } \\
\text { hạng tín dụng đối với khách hàng cá } \\
\text { nhân }\end{array}$ & & & & & \\
\hline $\begin{array}{l}\text { TTK } \\
\text { S4 }\end{array}$ & $\begin{array}{l}\text { Tính hiệu lực trong kiểm soát tín dụng } \\
\text { từ thực hiện theo quy định nội bộ về bảo } \\
\text { đảm nợ vay }\end{array}$ & & & & & \\
\hline $\begin{array}{l}\text { TTK } \\
\text { S5 }\end{array}$ & $\begin{array}{l}\text { Tính hiệu lực của cơ chế phê duyệt tín } \\
\text { dụng theo nguyên tắc kiểm soát "4 mắt" }\end{array}$ & & & & & \\
\hline $\begin{array}{l}\text { TTK } \\
\text { S6 }\end{array}$ & $\begin{array}{l}\text { Tính hiệu lực của cơ chế kiểm soát } \\
\text { chuyển nhóm nợ tự động được định } \\
\text { dạng trước trong hệ thống công nghệ } \\
\text { thông tin NH }\end{array}$ & & & & & \\
\hline $\begin{array}{l}\text { TTK } \\
\text { S7 }\end{array}$ & $\begin{array}{l}\text { Việc lưu trữ hồ sơ, chứng từ chỉ có } \\
\text { những người có thẩm quyền mới được } \\
\text { tiếp cận các thông tin này. }\end{array}$ & & & & & \\
\hline $\begin{array}{l}\text { TTK } \\
\text { S8 }\end{array}$ & $\begin{array}{l}\text { NH thực hiện các biện pháp để đảm bảo } \\
\text { an toàn cho thông tin trên hệ thống máy } \\
\text { tính, có hệ thống sao lưu phòng trường } \\
\text { hợp mất căp. }\end{array}$ & & & & & \\
\hline $\begin{array}{l}\text { TTK } \\
\text { S9 }\end{array}$ & $\begin{array}{l}\text { NH thường xuyên kiểm tra, đối chiếu } \\
\text { theo kế hoạch và đột xuất các hoạt động } \\
\text { tín dụng. }\end{array}$ & & & & & \\
\hline
\end{tabular}




\begin{tabular}{|c|c|c|c|c|c|c|}
\hline STT & Diễn giải & $\begin{array}{l}\text { (1) } \\
\text { Rất } \\
\text { thấp }\end{array}$ & $\begin{array}{l}\text { (2) } \\
\text { Thấp }\end{array}$ & $\begin{array}{l}\text { (3) } \\
\text { Trung } \\
\text { bình }\end{array}$ & $\begin{array}{l}\text { (4) } \\
\text { Cao }\end{array}$ & $\begin{array}{l}\text { (5) } \\
\text { Rất } \\
\text { cao }\end{array}$ \\
\hline TT1 & $\begin{array}{l}\text { Hệ thống báo cáo của NH được xây } \\
\text { dựng kịp thời, khoa học, đúng đối tượng }\end{array}$ & $\square$ & $\square$ & $\square$ & $\square$ & $\square$ \\
\hline TT2 & $\begin{array}{l}\text { Ban lãnh đạo ngân hàng luôn được cung } \\
\text { cấp kịp thời và đầy đủ các thông tin bên } \\
\text { trong và bên ngoài ngân hàng về hoạt } \\
\text { động tín dụng. }\end{array}$ & $\square$ & $\square$ & $\square$ & $\square$ & $\square$ \\
\hline TT3 & $\begin{array}{l}\text { Việc trao đổi thông tin giữa các cấp } \\
\text { được thực hiện qua mạng nội bộ. }\end{array}$ & $\square$ & $\square$ & $\square$ & $\square$ & $\square$ \\
\hline TT4 & $\begin{array}{l}\text { NH thực hiện cách thức để tiếp nhận ý } \\
\text { kiến khách hàng về vi phạm, sai sót của } \\
\text { cán bộ, nhân viên (hộp thư góp ý, đường } \\
\text { dây nóng, bộ phận chăm sóc khách } \\
\text { hàng...) }\end{array}$ & $\square$ & $\square$ & $\square$ & $\square$ & $\square$ \\
\hline TT5 & $\begin{array}{l}\text { Các quy định, chính sách TD nội bộ } \\
\text { được thông tin, truyền thông đến nhân } \\
\text { viên bằng văn bản kịp thời, rõ ràng, cụ } \\
\text { thể }\end{array}$ & $\square$ & $\square$ & $\square$ & $\square$ & $\square$ \\
\hline TT6 & $\begin{array}{l}\text { Các quy định, chính sách TD nội bộ } \\
\text { được thông tin, truyền thông đến nhân } \\
\text { viên được thể hiện dưới dạng bảng câu } \\
\text { hỏi và trả lời về nội dung cần kiểm soát } \\
\text { tín dụng rô ràng, cụ thể }\end{array}$ & $\square$ & $\square$ & $\square$ & $\square$ & $\square$ \\
\hline TT7 & $\begin{array}{l}\text { Thông tin truyền thông cảnh báo rủi ro } \\
\text { tín dụng đến lãnh đạo và nhân viên được } \\
\text { duy trì thường xuyên }\end{array}$ & $\square$ & $\square$ & $\square$ & $\square$ & $\square$ \\
\hline \multicolumn{7}{|c|}{ Nhân tố "Giám sát" - GS } \\
\hline GS1 & $\begin{array}{l}\text { NH thực hiện giám sát suốt quá trình } \\
\text { cho vay. }\end{array}$ & $\square$ & $\square$ & $\square$ & $\square$ & $\square$ \\
\hline GS2 & $\begin{array}{l}\text { NH thường xuyên gởi thư đối chiếu, } \\
\text { thăm dò ý kiến khách hàng vay }\end{array}$ & $\square$ & $\square$ & $\square$ & $\square$ & $\square$ \\
\hline
\end{tabular}




\begin{tabular}{|c|c|c|c|c|c|c|}
\hline STT & Diễn giải & $\begin{array}{l}\text { (1) } \\
\text { Rất } \\
\text { thấp }\end{array}$ & $\begin{array}{l}\text { (2) } \\
\text { Thấp }\end{array}$ & $\begin{array}{l}\text { (3) } \\
\text { Trung } \\
\text { bình }\end{array}$ & $\begin{array}{l}(4) \\
\text { Cao }\end{array}$ & $\begin{array}{l}\text { (5) } \\
\text { Rất } \\
\text { cao }\end{array}$ \\
\hline GS3 & $\begin{array}{l}\text { NH gặp gỡ trực tiếp, giải quyết khiếu } \\
\text { nại, thắc mắc của khách hàng, tư vấn } \\
\text { cho khách hàng }\end{array}$ & $\square$ & $\square$ & $\square$ & $\square$ & $\square$ \\
\hline GS4 & $\begin{array}{l}\text { NH có các biện pháp xử lý kịp thời đảm } \\
\text { bảo quyền lợi cho khách hàng. }\end{array}$ & $\square$ & $\square$ & $\square$ & $\square$ & $\square$ \\
\hline GS5 & $\begin{array}{l}\text { Chất lượng các cảnh báo rủi ro tín dụng } \\
\text { của Kiểm toán nội bộ, kiểm tra nội bộ } \\
\text { sau mỗi đợt kiểm toán }\end{array}$ & $\square$ & $\square$ & $\square$ & $\square$ & $\square$ \\
\hline GS6 & $\begin{array}{l}\text { Chất lượng của các báo cáo tự đánh giá, } \\
\text { tự chẩn chỉnh của NH về hoạt động tín } \\
\text { dụng }\end{array}$ & $\square$ & $\square$ & $\square$ & $\square$ & $\square$ \\
\hline GS7 & $\begin{array}{l}\text { Tính kịp thời của cảnh báo rủi ro tín } \\
\text { dụng trong ngân hàng của uỷ ban quản } \\
\text { lý tín dụng }\end{array}$ & $\square$ & $\square$ & $\square$ & $\square$ & $\square$ \\
\hline \multicolumn{7}{|c|}{ Nhân tố “Tính hũu hiệu của hệ thống kiểm soát nội bộ hoạt động tín dụng"-HH } \\
\hline HH1 & $\begin{array}{l}\text { Hệ thống KSNB của NH có tác động } \\
\text { tích cực trong ngăn ngừa, hạn chế rủi ro } \\
\text { tính dụng và nâng cao hiệu quả hoạt } \\
\text { động tín dụng. }\end{array}$ & $\square$ & $\square$ & $\square$ & $\square$ & $\square$ \\
\hline $\mathrm{HH} 2$ & $\begin{array}{l}\text { Mức độ thực hiện chỉ tiêu dư nợ tín } \\
\text { dụng so với kế hoạch }\end{array}$ & $\square$ & $\square$ & $\square$ & $\square$ & $\square$ \\
\hline $\mathrm{HH} 3$ & $\begin{array}{l}\text { Mức độ thực hiện chỉ tiêu nợ xấu so với } \\
\text { kế hoạch }\end{array}$ & $\square$ & $\square$ & $\square$ & $\square$ & $\square$ \\
\hline HH4 & $\begin{array}{l}\text { Mức độ thực hiện chỉ tiêu thu nhập lãi } \\
\text { so với kế hoạch }\end{array}$ & $\square$ & $\square$ & $\square$ & $\square$ & $\square$ \\
\hline
\end{tabular}

Nguồn: Kết quả phân tích dữ liệu của nhóm nghiên cứu

\section{Kết quả nghiên cứu và thảo luận}

Đặc trưng của mẫu nghiên cúu

Mẫu nghiên cứu gồm 250 quan sát trong đó có 149 nữ chiếm 59,6\% và 101 nam chiếm 40,4\%. Số quan sát thuộc NHTM Nhà nước (NHTM NN) chiếm 66,4\%, NHTM cổ phần (NHTM CP) chiếm 33,6\%, trình độ đại học trở lên chiếm $98,8 \%$. Phần lớn mẫu quan sát đều 
từ những người làm việc trực tiếp và gián tiếp liên quan đến hoạt động tín dụng với thâm niêm công tác từ 5 năm trở lên chiếm tỷ lệ $34 \%$, trong đó lãnh đạo chiếm tỷ lệ $21,6 \%$, nhân viên chiếm $78,4 \%$. Với kết quả phân tích như trên cho thấy mẫu nghiên cứu phân phối tương đối phù hợp.

\section{Kết quả phân tích thống kê mô tả}

\section{Bảng 4}

Giá trị trung bình các biến độc lập

\begin{tabular}{|c|c|c|c|c|c|c|c|c|c|c|}
\hline \multirow{2}{*}{ STT } & \multicolumn{90}{|c|}{ Tên biến và giá trị trung bình } \\
\cline { 2 - 13 } & \multicolumn{2}{|c|}{ MT } & \multicolumn{2}{|c|}{ ĐG } & \multicolumn{2}{c|}{ TTKS } & \multicolumn{2}{|c|}{ TT } & \multicolumn{2}{|c|}{ GS } \\
\hline 1 & MT1 & 3,7560 & ĐG1 & 3,4920 & TTKS1 & 4,1640 & TT1 & 3,6240 & GS1 & 3,5840 \\
\hline 2 & MT2 & 3,7440 & ĐG2 & 3,4040 & TTKS2 & 4,0040 & TT2 & 3,9720 & GS2 & 3,1560 \\
\hline 3 & MT3 & 3,6480 & ĐG3 & 3,5920 & TTKS3 & 3,6200 & TT3 & 3,8440 & GS3 & 3,6400 \\
\hline 4 & MT4 & 3,7360 & ĐG4 & 3,6880 & TTKS4 & 3,8440 & TT4 & 3,8720 & GS4 & 3,6680 \\
\hline 5 & MT5 & 3,5640 & ĐG5 & 3,6560 & TTKS5 & 3,9280 & TT5 & 3,9200 & GS5 & 3,7080 \\
\hline 6 & MT6 & 3,9760 & & & TTKS6 & 3,8160 & TT6 & 3,3360 & GS6 & 3,5680 \\
\hline 7 & MT7 & 3,7400 & & & TTKS7 & 3,9880 & TT7 & 3,6680 & GS7 & 3,5360 \\
\hline 8 & MT8 & 3,3800 & & & TTKS8 & 3,7400 & & & & \\
\hline 9 & MT9 & 3,6800 & & & TTKS9 & 3,8240 & & & & \\
\hline TB & MT & $\mathbf{3 , 6 9 1 6}$ & ĐG & $\mathbf{3 , 5 6 6 4}$ & TTKS & $\mathbf{3 , 8 8 0 9}$ & TT & $\mathbf{3 , 7 4 8 0}$ & GS & $\mathbf{3 , 5 5 1 4}$ \\
\hline
\end{tabular}

Nguồn: Kết quả phân tích dữ liệu của nhóm nghiên cứu

Kết quả phân tích thống kê mô tả trong Bảng 4 cho thấy thang đo MT được đánh giá ở mức trung bình so với các thang đo khác, mức trung bình là 3,6916 trong thang đo Likert 5 điểm. Biến quan sát được đánh giá cao nhất là biến MT6: "NH có quy định cu thể và thể chế hóa bằng văn bản rõ ràng chí̛c năng, trách nhiệm của cán bộ quản lí và tùng nhân viên" với giá trị trung bình là 3,9760. Kế đến là các biến quan sát $M T 1, M T 2$ và $M T 7$ đều có mức trung bình lớn hơn 3,7400. Biến quan sát được đánh giá thấp nhất trong thang đo này là biến $M T 8$ : "Bộ phận kiểm soát nội bộ thực hiện được chức năng kiểm soát rủi ro tín dụng tại ngân hàng" với mức trung bình là 3,3800 .

Thang đo ĐG với giá trị trung bình là 3,5664 là thang đo có giá trị trung bình thấp thứ hai, sau thang đo GS. Cụ thể thang đo ĐG có biến quan sát ĐG4: "Mức độ linh hoạt về lãi suất tín dụng đối với khách hàng trên cơ sở phân loại khách hàng khi xét cấp tín dụng" được đánh giá cao nhất trong thang đo này với giá trị trung bình là 3,6880. Kế đến là biến ĐG5: "Ngân hàng có tiêu chí cảnh báo sớm nợ có vấn đề" với mức trung bình là 3,6560. Tuy nhiên biến quan sát ĐG2: "Tính kịp thời của thông tin cảnh báo rủi ro khi có sụ thay đổi bất lợi trong môi truờng kinh doanh hoạt động tín dụng" được đánh giá thấp nhất với mức trung bình là 3,4040. 
Thang đo TTKS là thang đo được đánh giá cao nhất với mức trung bình là 3,8809 trong thang đo Likert 5 điểm và cao nhất trong các biến độc lập còn lại. Trong thang đo biến TTKS cho thấy các biến quan sát TTKS1: "Tính hiệu lực trong kiểm soát tín dụng tù thưc hiện theo quy trình tín dụng nội bộ $N H$ ” được đánh giá ở mức cao nhất với giá trị trung bình là 4,1640, kế đến là biến TTKS2: "Tính hiệu lục trong kiểm soát tín dụng tù thực hiện theo quy định nội bộ về xếp hạng tín dụng đối với khách hàng doanh nghiệp” với giá trị trung bình 4,0040, đứng thứ ba là biến TTKS7: "Việc lưu trũu hồ so", chứng tù chi có nhũng người có thẩm quyền mới được tiếp cận các thông tin này” với mức trung bình là 3,988. Biến quan sát TTKS5: "Tính hiệu lực của co chế phê duyệt tín dụng theo nguyên tắc kiểm soát ít nhất thông qua 2 ngườ" cũng được đánh giá cao ở mức trung bình là 3,9280 so với các biến quan sát còn lại. Biến quan sát được đánh giá thấp nhất là biến TTKS3: "Tỉnh hiệu lực trong kiểm soát tín dụng tù thực hiện theo quy định nội bộ về xếp hạng tín dụng đối với khách hàng cá nhân" với mức trung bình là 3,6200 điểm, kế đến là biến TTKS8: "NH thưc hiện các biện pháp để đảm bảo an toàn cho thông tin trên hệ thống máy tính, có hệ thống sao lưu phòng trương hợp mất cắp" với giá trị trung bình đạt được là 3,7400.

Thang đo TT được đánh giá cao thứ hai so với các thang đo khác, mức trung bình là 3,7480. Biến quan sát được đánh giá thấp nhất trong thang đo này là biến TT6: "Các quy định chính sách tín dụng nội bộ được thông tin truyền thông đến nhân viên và thể hiện dưới dạng câu hỏi, câu trả lời về nội dung cần kiểm soát tín dụng rõ ràng cu thể,", với mức trung bình là 3,3360. Biến quan sát được đánh giá cao nhất là TT2: "Ban lãnh đạo ngân hàng luôn được cung cấp kịp thời và đầy đủ các thông tin bên trong và bên ngoài ngân hàng về hoạt động tín dụng" với giá trị trung bình là 3,9720.

Thang đo GS là thang đo được đánh giá thấp nhất trong các thang đo của các biến độc lập, với mức trung bình là 3,5514. Trong đó biến quan sát được đánh giá thấp nhất là $G S 2$ : "Ngân hàng thương xuyên gưi thu đối chiếu thăm dò ý kiến khách hàng vay" với giá trị trung bình là 3,1560. Biến quan sát được đánh giá thấp kế tiếp là GS7: "Tính kịp thời của cảnh báo rủi ro tín dụng trong ngân hàng của uỷ ban quản lí tín dụng", với giá trị trung bình bằng 3,5360. Hai biến quan sát được đánh giá cao nhất trong thang đo này là biến GS5: "Chất lương các cảnh báo rủi ro tín dụng của Kiểm toán nội bộ, kiểm tra nội bộ sau mỗi đợt kiểm toán" với mức trung bình là 3,7080 và biến GS4: "NH có các biện pháp xủ lí kịp thời đảm bảo quyền lợi cho khách hàng” với mức trung bình là 3,6680.

Đối với thang đo biến phụ thuộc $(\mathrm{HH})$, kết quả phân tích Bảng 4 cho thấy mức đánh giá trung bình tương đối đồng đều giữa các biến quan sát với giá trị trung bình của thang đo là 3,7026. Chỉ có biến HH3: Mưc độ thực hiện chi tiêu nợ xấu so với kế hoạch được đánh giá thấp nhất với trung bình là 3,5771 .

\section{Bảng 5}

Thống kê mô tả biến phụ thuộc

\begin{tabular}{|c|c|}
\hline Tên biến & Trung bình \\
\hline HH1 & 3,7391 \\
\hline
\end{tabular}




\begin{tabular}{|c|c|}
\hline Tên biến & Trung bình \\
\hline HH2 & 3,7154 \\
\hline HH3 & 3,5771 \\
\hline HH4 & 3,7787 \\
\hline HH & 3,7026 \\
\hline
\end{tabular}

Nguồn: Kết quả phân tích dữ liệu của nhóm nghiên cứu

\section{Kết quả phân tích độ tin cậy của các thang đo}

Thang đo MT: Có 9 biến quan sát với Cronbach's Alpha của thang đo là 0,880 . Các biến quan sát đều có hệ số tương quan biến tổng lớn hơn 0,3 và Cronbach's Alpha nhỏ hơn 0,880 .

Thang đo ĐG: Có 5 biến quan sát và Cronbach's Alpha của thang đo là 0,835 . Các biến quan sát đều có hệ số tương quan biến tổng lớn hơn 0,3 và Cronbach's Alpha nhỏ hơn 0,835 .

Thang đo TTKS: Có 9 biến quan sát với Cronbach's Alpha của thang đo là 0,899 . Các biến quan sát đều có hệ số tương quan biến tổng lớn hơn 0,3 và và Cronbach's Alpha nhỏ hơn 0,899 .

Thang đo TT: Có 7 biến quan sát với Cronbach's Alpha của thang đo là 0,865 . Các biến quan sát đều có hệ số tương quan biến tổng lớn hơn 0,3 và và Cronbach's Alpha nhỏ hơn 0,865 .

Thang đo GS: Có 7 biến quan sát với Cronbach's Alpha của thang đo là 0,859 . Các biến quan sát đều có hệ số tương quan biến tổng lớn hơn 0,3 và Cronbach's Alpha nhỏ hơn 0,859 .

Thang đo biến phụ thuộc HH: Có 4 biến quan sát và Cronbach’s Alpha của thang đo là 0,784 . Các biến quan sát đều có hệ số tương quan biến tổng lớn hơn 0,3 và Cronbach's Alpha nhỏ hơn 0,784 .

Kết quả phân tích cho thấy thang đo các biến độc lập và biến phụ thuộc đều đạt yêu cầu về độ tin cậy.

\section{Phân tích nhân tố khám phá:}

Các thang đo sau khi được kiểm định độ tin cậy và đạt yêu cầu lần lượt được đưa vào phân tích nhân tố khám phá. Nghiên cứu sử dụng phương pháp rút trích Principal Axis Factoring với phép xoay Promax. Kết quả cho thấy sau khi phân tích nhân tố đã rút trích được 5 nhân tố với phương sai trích đạt được là 56,53\%. Hệ số KMO của kiểm định Bartlett bằng 0,925 và giá trị Sig của phép kiểm định $=0,000$. Các hệ số tải nhân tố đều lớn hơn 0,4 . Điều này cho thấy phân tích nhân tố là phù hợp. Kết quả được thể hiện trong Bảng 6 . 


\section{Bảng 6}

Kết quả phân tích nhân tố khám phá

\begin{tabular}{|c|c|c|c|c|c|}
\hline & & & Nhân t' & & \\
\hline $\begin{array}{c}\text { Biến quan } \\
\text { sát }\end{array}$ & 1 & 2 & 3 & 4 & 5 \\
\hline MT1 & 0,897 & & & & \\
\hline MT2 & 0,677 & & & & \\
\hline MT3 & 0,876 & & & & \\
\hline MT4 & 0,628 & & & & \\
\hline MT5 & 0,647 & & & & \\
\hline DG1 & & 0,631 & & & \\
\hline DG2 & & 0,796 & & & \\
\hline DG3 & & 0,672 & & & \\
\hline DG5 & & 0,547 & & & \\
\hline TTKS1 & & & 0,655 & & \\
\hline TTKS2 & & & 0,663 & & \\
\hline TTKS4 & & & 0,744 & & \\
\hline TTKS5 & & & 0,701 & & \\
\hline TTKS6 & & & 0,699 & & \\
\hline TTKS7 & & & 0,791 & & \\
\hline TTKS8 & & & 0,660 & & \\
\hline TT2 & & & & 0,967 & \\
\hline TT3 & & & & 0,710 & \\
\hline TT4 & & & & 0,707 & \\
\hline TT5 & & & & 0,589 & \\
\hline TT6 & & & & & 0,579 \\
\hline GS2 & & & & & 0,768 \\
\hline GS3 & & & & & 0,721 \\
\hline GS4 & & & & & 0,626 \\
\hline GS5 & & & & & 0,654 \\
\hline GS6 & & & & & 0,586 \\
\hline GS7 & & & & & 0,615 \\
\hline
\end{tabular}

Nguồn: Kết quả phân tích dữ liệu của nhóm nghiên cứu

Sau khi phân tích nhân tố, các thang đo bao gồm: 


\section{Bảng 7}

Nhân tố 1 (Môi trường kiểm soát) bao gồm các biến: MT1, MT2, MT3, MT4, MT5

\begin{tabular}{|l|l|}
\hline Kí hiệu & \multicolumn{1}{|c|}{ Môi trường (MT) } \\
\hline MT 1 & Lãnh đạo NH chấp hành tốt các quy định kiểm soát tín dụng tại ngân hàng \\
\hline MT2 & NH có chính sách tuyển dụng nhân viên và nhân viên tín dụng rõ ràng. \\
\hline MT3 & NH có kế hoạch cụ thể, rõ ràng chính sách đào tạo đối với lãnh đạo và nhân viên. \\
\hline MT4 & NH có chính sách lương, thưởng, kỷ luật rõ ràng, cụ thể \\
\hline MT5 & NH có chính sách cụ thể vể luân chuyển cán bộ, nhân viên theo định kì, \\
\hline
\end{tabular}

Nguồn: Kết quả phân tích dữ liệu của nhóm nghiên cứu

\section{Bảng 8}

Nhân tố 2 (Đánh giá rủi ro) gồm các biến: DG1, DG2, DG3, DG5

\begin{tabular}{|l|l|}
\hline Kí hiệu & \multicolumn{1}{|c|}{ ĐÁNH GIÁ (DG) } \\
\hline ĐG1 & $\begin{array}{l}\text { Quy trình soát xét chất lượng tín dụng có khả năng dự báo sớm những thay đổi về } \\
\text { tình hình tài chính, khả năng trả nợ của các bên đối tác }\end{array}$ \\
\hline ĐG2 & $\begin{array}{l}\text { Tính kịp thời của các thông tin cảnh báo rủi ro khi có sự thay đổi bất lợi trong môi } \\
\text { trường kinh doanh, hoạt động tín dụng }\end{array}$ \\
\hline ĐG3 & Tính cập nhật của các quy định về ngành nghề kinh doanh, quản lí rủi ro tín dụng \\
\hline ĐG5 & NH có các tiêu chí cảnh báo sớm nợ có vấn đề \\
\hline
\end{tabular}

Nguồn: Kết quả phân tích dữ liệu của nhóm nghiên cứu

\section{Bảng 9}

Nhân tố 3 (Thủ tục kiểm soát) bao gồm các biến: MT6, TTKS1, TTKS2, TTKS4, TTKS5, TTKS6, TTKS7, TTKS8

\begin{tabular}{|l|l|}
\hline Kí hiệu & \multicolumn{1}{c|}{ THỦ TỤC KIỂM SOÁT (TTKS) } \\
\hline TTKS1 & Tính hiệu lực trong kiểm soát tín dụng từ thực hiện theo quy trình tín dụng NH. \\
\hline TTKS2 & $\begin{array}{l}\text { Tính hiệu lực trong kiểm soát tín dụng từ thực hiện theo quy định về xếp hạng tín } \\
\text { dụng đối với khách hàng }\end{array}$ \\
\hline TTKS4 & $\begin{array}{l}\text { Tính hiệu lực trong kiểm soát tín dụng từ thực hiện theo quy định về bảo đảm nợ } \\
\text { vay }\end{array}$ \\
\hline TTKS5 & Tính hiệu lực của cơ chế phê duyệt tín dụng theo nguyên tắc kiểm soát “4 mắt” \\
\hline TTKS6 & $\begin{array}{l}\text { Tính hiệu lực của cơ chế kiểm soát chuyển nhóm nợ tự động được định dạng } \\
\text { trước trong hệ thông công nghệ thông tin NH }\end{array}$ \\
\hline
\end{tabular}




\begin{tabular}{|c|l|}
\hline Kí hiệu & \multicolumn{1}{|c|}{ THỦ TỤC KIỂM SOÁT (TTKS) } \\
\hline TTKS7 & $\begin{array}{l}\text { Việc lưu trữ hồ sơ, chứng từ chỉ có những người có thẩm quyền mới được tiếp } \\
\text { cận các thông tin này. }\end{array}$ \\
\hline TTKS8 & $\begin{array}{l}\text { NH thực hiện các biện pháp để đảm bảo an toàn cho thông tin trên hệ thống máy } \\
\text { tính, có hệ thống sao lưu phòng trường hợp mất cắp. }\end{array}$ \\
\hline
\end{tabular}

Nguồn: Kết quả phân tích dữ liệu của nhóm nghiên cứu

\section{Bảng 10}

Nhân tố 4 (Thông tin và truyền thông) bao gồm các biến: TT3, TT4, TT5, TT6

\begin{tabular}{|l|l|}
\hline Kí hiệu & \multicolumn{1}{|c|}{ THÔNG TIN VÀ TRUYỀ THÔNG (TT) } \\
\hline TT2 & $\begin{array}{l}\text { Ban lãnh đạo ngân hàng luôn được cung cấp kịp thời và đầy đủ các thông tin bên } \\
\text { trong và bên ngoài ngân hàng về hoạt động tín dụng. }\end{array}$ \\
\hline TT3 & Việc trao đổi thông tin giữa các cấp được thực hiện qua mạng nội bộ. \\
\hline TT4 & $\begin{array}{l}\text { NH thực hiện cách thức để tiếp nhận ý kiến khách hàng về vi phạm, sai sót của cán } \\
\text { bộ, nhân viên (hộp thư góp ý, đường dây nóng, bộ phận chăm sóc khách hàng...) }\end{array}$ \\
\hline TT5 & $\begin{array}{l}\text { Các quy định, chính sách TD nội bộ được thông tin, truyền thông đến nhân viên } \\
\text { bằng văn bản kịp thời, rõ ràng, cụ thể }\end{array}$ \\
\hline
\end{tabular}

Nguồn: Kết quả phân tích dữ liệu của nhóm nghiên cứu

\section{Bảng 11}

Nhân tố 5 (Giám sát) bao gồm các biến: GS2, GS3, GS4, GS5, GS6, GS7

\begin{tabular}{|l|l|}
\hline Kí hiệu & GIÁM SÁT (GS) \\
\hline GS2 & NH thường xuyên gởi thư đối chiếu, thăm dò ý kiến khách hàng vay \\
\hline GS3 & $\begin{array}{l}\text { NH gặp gỡ trực tiếp, giải quyết khiếu nại, thắc mắc của khách hàng, tư vấn cho } \\
\text { khách hàng }\end{array}$ \\
\hline GS4 & NH có các biện pháp xử lí kịp thời đảm bảo quyền lợi cho khách hàng. \\
\hline GS5 & $\begin{array}{l}\text { Chất lượng các cảnh báo rủi ro tín dụng của kiểm toán nội bộ, kiểm tra nội bộ sau } \\
\text { mổi đợt kiểm toán }\end{array}$ \\
\hline GS6 & $\begin{array}{l}\text { Chất lượng của các báo cáo tự đánh giá, tự chấn chỉnh của NH về hoạt động tín } \\
\text { dụng }\end{array}$ \\
\hline GS7 & Tính kịp thời của cảnh báo rủi ro tín dụng trong ngân hàng \\
\hline
\end{tabular}

Nguồn: Kết quả phân tích dữ liệu của nhóm nghiên cứu

Kiểm định lại độ tin cậy của các thang đo sau khi phân tích nhân tố khám phá cho thấy đều đạt yêu cầu. 


\section{Kết quả phân tích hồi quy}

Sau khi các thang đo đều đạt yêu cầu về độ tin cậy, nghiên cứu tiếp tục thực hiện phân tích hồi quy theo phương pháp bình phương bé nhất (OLS) để xem xét mức độ tác động giữa các biến độc lập đến biến phụ thuộc. Kết quả phân tích hồi quy được thể hiện trong Bảng 7 .

\section{Bảng 12}

Kết quả phân tích hồi quy theo phương pháp OLS

\begin{tabular}{|l|l|l|l|}
\hline \multicolumn{1}{|c|}{ Tên biến } & \multicolumn{1}{c|}{ Hệ số $\boldsymbol{\beta}$} & \multicolumn{1}{c|}{ Hệ số $\boldsymbol{\beta}$ chuẩn hóa } & \multicolumn{1}{c|}{ Giá trị P - value } \\
\hline Hằng số & 0,281 & & 0,141 \\
\hline MT & 0,109 & 0,124 & 0,022 \\
\hline ĐG & 0,062 & 0,064 & 0,275 \\
\hline TTKS & 0,308 & 0,290 & 0,000 \\
\hline TT & 0,097 & 0,114 & 0,038 \\
\hline GS & 0,351 & 0,330 & 0,000 \\
\hline $\begin{array}{l}\text { Giá trị Sig. của mô hình }=0,000 \\
\text { R }=58,2 \% \\
\text { Hệ số Durbin-watson }=1,725 \\
\text { Hệ số VIF < 10 }\end{array}$ & \\
\hline
\end{tabular}

Nguồn: Kết quả phân tích dữ liệu của nhóm nghiên cứu

Với kết quả hồi quy trong Bảng 7 cho thấy mô hình hồi quy có giá trị Sig. $=0,000$ nhỏ hơn mức ý nghĩa $5 \%$ nên mô hình hồi quy là có ý nghĩa thống kê. Giá trị $\mathrm{R}^{2}=58,2 \%$ cho thấy các biến độc lập trong mô hình giải thích được $58,2 \%$ sự biến thiên của biến phụ thuộc. Hệ số Durbin - watson $=1,725$ gần bằng 2 và các hệ số VIF đều nhỏ hơn 10 nên mô hình hồi quy không xảy ra hiện tượng tự tương quan và đa cộng tuyến.

Kết quả phương trình hồi quy thể hiện như sau:

$$
\mathrm{HH}=0,281+0,109 * \mathrm{MT}+0,308 * \mathrm{TTKS}+0,097 * \mathrm{TT}+0,351 * \mathrm{GS}+\varepsilon
$$

Trong 5 biến độc lập thì 4 biến MT, TTKS, TT và GS tác động lên biến HH ở mức ý nghĩa 5\%. Trong mẫu nghiên cứu sự tác động của biến ĐG đến $\mathrm{HH}$ là không có ý nghĩa thống kê. Như vậy mô hình hồi quy cho thấy bốn thành phần MT, TTKS, TT và GS đều tác động tích cực lên biến $\mathrm{HH}$, trong đó tác động của biến GS là mạnh nhất thể hiện ở hệ số $\beta$ chuẩn hóa $=$ 0,330 và kế đến là biến TTKS với $\beta$ chuẩn hóa $=0,290$ và biến $\mathrm{MT}$ với $\beta$ chuẩn hóa $=0,124$ và cuối cùng là TT với $\beta$ chuẩn hóa $=0,114$. 


\section{Kết luận và khuyến nghị}

Kết quả nghiên cứu cho thấy phần lớn các nhân tố (biến độc lập) như MT, TT, TTKS và GS (ngoại trừ ĐG) đều có ảnh hưởng đến (biến phụ thuộc) tính hữu hiệu của KSNB hoạt động tín dụng tại NHTM VN, thể hiện trong kết quả phương trình hồi quy:

$$
\mathrm{HH}=0,281+0,109 * \mathrm{MT}+0,308 * \mathrm{TTKS}+0,097 * \mathrm{TT}+0,351 * \mathrm{GS}+\varepsilon
$$

Kết quả phân tích cho thấy có 4 thành phần gồm Môi trường kiểm soát, Thủ tục kiểm soát, Giám sát và Thông tin và truyền thông tác động tích cực lên Tính hữu hiệu của KSNB hoạt động tín dụng ngân hàng. Mức độ tác động mạnh nhất thuộc về thành phần Giám sát, kế đến là thành phần Thủ tục kiểm soát, tiếp theo là thành phần Môi trường kiểm soát và cuối cùng là thành phần Thông tin và truyền thông.

Căn cứ vào phương trình hồi quy, khi thành phần Giám sát tăng lên 1 thì tính hữu hiệu của KSNB hoạt động tín dụng tăng lên 0,351 . Hoàn toàn tương tự khi các thành phần Thủ tục kiểm soát, Môi trường kiểm soát và Thông tin và truyền thông tăng lên 1 thì tính hữu hiệu của KSNB hoạt động tín dụng tăng lên lần lượt là 0,$308 ; 0,109$ và 0,097 .

Trong mẫu nghiên cứu, thành phần Đánh giá rủi ro tác động đến tính hữu hiệu của KSNB hoạt động tín dụng là không có ý nghĩa thống kê. Nhìn vào kết quả phân tích ở Bảng 4 , có thể thấy thành phần đánh giá (ĐG) với giá trị trung bình là 3,5664 là thang đo có giá trị trung bình thấp thứ hai trong các thang đo của các biến độc lập, trong đó biến quan sát $Đ G 2$ "Tính kịp thời của thông tin cảnh báo rủi ro khi có sư thay đổi bất lợi trong môi trường kinh doanh hoạt động tín dụng" được đánh giá thấp nhất với mức trung bình là 3,4040.

So sánh với nghiên cứu của Ayagre và cộng sự (2014), tiếp cận 5 nhân tố cấu thành HTKSNB theo COSO (2013), kết quả HTKSNB tại các NH ở Ghanaian tương đối tốt, nhân tố môi trường kiểm soát và giám sát hoạt động được đánh giá cao, điểm trung bình 4,72 và 4,66 , kết quả nghiên cứu của bài viết này cho thấy các nhân tố ảnh hưởng đến tính hữu hiệu HTKSNB tín dụng tại 26 NHTM Việt Nam trong mẫu khảo sát chỉ được đánh giá trên mức trung bình từ 3,5514 đến 3,8809 (Bảng 3), điều này là cơ sở đáng lưu ý để các NHTM cần có những giải pháp nâng cao tính hữu hiệu của HTKSNB hoạt động tín dụng.

Dựa trên kết quả nghiên cứu và khảo sát ý kiến đúc kết thông qua câu hỏi mở từ lãnh đạo và nhân viên NHTM Việt Nam, bài viết đề xuất các gợi ý giải pháp để nâng cao tính hữu hiệu trong KSNB hoạt động tín dụng tại các NHTM Việt Nam như sau:

Về môi trường kiểm soát: NHTM cần tăng cường phổ biến, nhắc lại cẩm nang đạo đức và yêu cầu kí cam kết thực hiện hàng năm, thực hiện luân chuyển cán bộ và nhân viên định kì để hạn chế rủi ro tác nghiệp, rủi ro vi phạm đạo đức nghề nghiệp do quản lí chi nhánh, quản lí khách hàng quá lâu, quá quen thuộc. Nhân sự của bộ phận kiểm toán nội bộ cần được tuyển chọn có năng lực cao và có sự thay đổi (định kì 3 năm/ lần) để phát huy được chức năng tư vấn tạo giá trị gia tăng trong báo cáo kiểm toán, không nên chỉ dừng lại trong báo cáo phát hiện lỗi sai sót trong tín dụng.

Về giám sát rủi ro: Hoạt động giám sát rủi ro bao gồm hoạt động tự giám sát thường xuyên của lãnh đạo và nhân viên thông qua thực hiện các quy định về tín dụng và giám sát độc 
lập từ bộ phận kiểm toán nội bộ. Vì vậy, NHTM cần chú trọng xây dựng yêu cầu nhân viên thực hiện báo cáo bất thường về hoạt động và xử lí kịp thời các báo cáo bất thường. Ngân hàng cần nâng cao chất lượng giám sát tín dụng thông qua yêu cầu xây dựng kế hoạch kiểm toán hàng năm một cách linh hoạt trong từng thời kì định hướng theo rủi ro. Kiểm toán viên nội bộ cũng phải thay đổi phương pháp kiểm toán, cần kết hợp phương pháp kiểm toán hệ thống với kiểm toán chi tiết nhằm đánh giá tính hiệu lực và hiệu quả của hoạt động tín dụng một cách toàn diện, nhờ đó kiểm toán viên sẽ tiết kiệm được thời gian kiểm toán nhưng lại có thể đưa ra kết luận tổng thể, định hướng vào rủi ro trong hoạt động tín dụng.

Về thủ tục kiểm soát: NHTM cần nâng cao tính hiệu lực của quy định về xếp hạng tín dụng nội bộ với mục tiêu sử dụng quy định này trong xem xét quyết định tín dụng, ưu đãi tín dụng, phân loại nợ và lập dự phòng rủi ro. NHTM cần xây dựng các quy trình tín dụng cụ thể đối với sản phẩm tín dụng, bổ sung các lưu ý rủi ro cần tăng cường kiểm soát đối với từng loại sản phẩm tín dụng, thực hiện tốt quy định về đảm bảo nợ vay cũng như nguyên tắc kiểm soát "4 mắt" trong phê duyệt tín dụng, nghĩa là trong quy trình tín dụng ít nhất phải thực hiện kiểm soát thông qua người thứ hai. Thủ tục kiểm soát thông qua giao chỉ tiêu tín dụng cần có căn cứ thực tế hạn chế mở rộng quy mô tín dụng chạy theo lợi nhuận xem nhẹ kiểm soát rủi ro dẫn đến nợ xấu tăng cao.

Về thông tin và truyền thông: NHTM cần đa dạng các hình thức truyền thông thông tin đến nhân viên và bằng nhiều hình thức khác nhau. Bên cạnh trao đổi thông tin bằng văn bản là chủ yếu như hiện nay, NHTM cần tăng cường xây dựng hệ thống báo cáo kịp thời, khoa học và đầy đủ các thông tin bên trong và bên ngoài ngân hàng về hoạt động tín dụng, trao đổi thông tin qua mạng nội bộ, tiếp nhận ý kiến khách hàng về vi phạm, sai sót của cán bộ, nhân viên thông qua hộp thư góp ý, đường dây nóng, bộ phận chăm sóc khách hàng. Các quy định, chính sách TD nội bộ cần được thông tin, truyền thông đến nhân viên được thể hiện dưới dạng lưu đồ phân quyền kiểm soát tín dụng, dưới dạng bảng câu hỏi và trả lời về nội dung cần kiểm soát tín dụng rõ ràng, cụ thể. Thông tin truyền thông cảnh báo rủi ro tín dụng đến lãnh đạo và nhân viên cần được duy trì thường xuyên.

Về đánh giá rủi ro: Tuy kết quả mô hình về nhân tố đánh giá rủi ro không có ý nghĩa thống kê nhưng kết quả khảo sát cũng cho thấy $\mathrm{NH}$ cần lưu ý thông tin cảnh báo rủi ro khi có sự thay đổi bất lợi trong môi trường kinh doanh, hoạt động tín dụng, xây dựng tiêu chí báo cáo và yêu cầu báo cáo cảnh báo sớm nợ vay có vấn đề.

Phân tích nhân tố bị giới hạn số lượng mẫu 250 mẫu từ lãnh đạo và nhân viên của 26 NHTM tại VN nên ý nghĩa thống kê và độ tin cậy của một số nhân tố tác động đến tính hiệu lực của KSNB về hoạt động tín dụng tại các NHTM còn có những hạn chế. Bài nghiên cứu tạo tiền đề cho các nghiên cứu tiếp theo trong việc mở rộng số lượng mẫu nhằm tăng mức ý nghĩa và độ tin cậy của mô hình nghiên cứu. Một số đề xuất để nâng cao tính hữu hiệu KSNB hoạt động tín dụng tại các NHTM trong phạm vi đề tài có liên quan đến các nhân tố ảnh hưởng đến KSNB theo mô hình của COSO cũng tạo tiền đề để mở rộng phạm vi các hướng đề xuất cho các nghiên cứu tiếp theo. 


\section{Tài liệu tham khảo}

Abiola, I., \& Oyewole, A. T. (2013). Internal control system on fraud detection: Nigeria experience. Journal of Accounting and Finance, 13(5), 141-152.

Ajala, O. A., Amuda, T., \& Arulogun, L. (2013). Evaluating internal control system as preventive measure of fraud in the Nigerian banking sector. Retrieved Jannuary 24, 2018, from https://zenodo.org/record/3441639\#.YEg5VB9MQ2w

Ayagre, P., Appiah-Gyamerah, I., \& Nartey, J. (2014). The effectiveness of internal control systems of banks. The case of Ghanaian banks. International Journal of Accounting and Financial Reporting, 4(2), 377-389.

Barakat, A. (2009). Banks Basel II norms requirement regarding internal control - Field study on Jordan banks, Delhi Business Review, 10(2), 35-48.

Basel (1998). Framework for internal control systems in banking organization. Retrieved Jannuary 27, 2016, from https://www.bis.org/publ/bcbsc131.pdf

COSO. (1992). Internal control - Integrated framework. Retrieved Jannuary 22, 2018, from http://www.developmentwork.net/projects/risk-management-standards/137-coso-1992internal-control-integrated-framework

COSO. (2013). Internal control - Integrated framework: Executive summary. Retrieved Jannuary 20, 2018, from https://na.theiia.org/standardsguidance/topics/documents/executive_summary.pdf

Dao, P. M., \& Le, H. V. (2012). HTKSNB gắn với quản lí rủi ro tại các NHTM VN trong giai đoạn hiện nay [Internal control system associated with risk management at Vietnamese commercial banks in the current period]. Tạp chí Ngân hàng, 24, 20-26.

Ngo, P. T., \& Le, N. T. T. (2015). Khuôn khổ HTKSNB theo tiêu chuẩn Basel [The framework of internal control system according to Basel standards]. Thị trường Tài chính Tiền tệ, 5, $18-21$.

Nguyen, P. A., \& Ha, H. T. T. (2010). Nâng cao hiệu quả hoạt động KSNB ở các NHTMCP trên địa bàn TP.HCM [Improve efficiency of internal control activities at commercial banks in Ho Chi Minh City]. Phát triển kinh tế, 240, 41-48.

Nguyen, P. M. (2014). Một số yếu kém trong quy trình KSNB hoạt động tín dụng của các NHTM và khuyến nghị [Some weaknesses in the process of internal control of credit operations of commercial banks and recommendations]. Tạp chí Ngân hàng, 6, 26-30.

Olatunji, O. C. (2009). Impact of internal control system in banking sector in Nigeria. Pakistan Journal of Social Sciences, 6(4), 181-189.

Pham, H. T. V., \& Nguyen, P. K. (2015). Yếu kém trong KSNB ở các NHTM VN [Weakness in internal control in Vietnamese commercial banks]. Kinh tế và dụ báo, 11, 27-29.

Salehi, M., Shiri, M. M., \& Ehsanpour, F. (2013). Effectiveness of internal control in the banking sector: Evidence from bank Mellat, Iran. IUP Journal of Bank Management, 12(1), 23-34. 
Sultana, R., \& Haque, M. E. (2011). Evaluation of internal control structure: Evidence from six listed banks in Banglades. ASA University Review, 5(1), 69-81.

Vo, N. T. H. (2015). Xây dựng mô hình KSNB hiệu quả, hiệu lực tại NHTM theo mô hình Coso 2013 [Building an effective and effective internal control model at commercial banks according to the 2013 Coso model]. Thị trường Tài chính Tiền tệ, 8, 38-43.

Vo, N. T. H., \& Le, H. T. T. (2014). Hoàn thiện HTKSNB của các NHTM VN theo mô hình Coso [Completing the internal control system of Vietnamese commercial banks according to the Coso model]. Tạp chí Ngân hàng, 4, 22-27. 